Mon. Not. R. Astron. Soc. 000, 1-?? (2013) Printed 22 March $2021 \quad$ (MN $\mathrm{IT}_{\mathrm{E}} \mathrm{X}$ style file v2.2)

\title{
Probing the surface magnetic field structure in RX J1856.5-3754
}

\author{
S. B. Popov ${ }^{1 \star}$, R. Taverna ${ }^{2}$, R. Turolla ${ }^{2,3}$ \\ ${ }^{1}$ Sternberg Astronomical Institute, Lomonosov Moscow State University, Moscow, Russia 119234 \\ ${ }^{2}$ Department of Physics and Astronomy, University of Padova, via Marzolo 8, 35131 Padova, Italy \\ ${ }^{3}$ Mullard Space Science Laboratory, University College London, Holbury St. Mary, Surrey, RH5 6NT, UK
}

Accepted .... Received ...; in original form ...

\begin{abstract}
The evolution of magnetic field in isolated neutron stars is one of the most important ingredients in the attempt to build a unified description of these objects. A prediction of field evolution models is the existence of an equilibrium configuration, in which the Hall cascade vanishes. Recent calculations have explored the field structure in this stage, called the Hall attractor. We use X-ray data of near-by, cooling neutron stars to probe this prediction, as these sources are surmised to be close to or at Hall attractor phase. We show that the source RX J1856.5-3754 might be closer to the attractor than other sources of its class. Our modelling indicates that the properties of surface thermal emission, assuming that the star is in the Hall attractor, are in contradiction with the spectral data of RX J1856.5-3754.
\end{abstract}

Key words: Magnetic fields - Radiation mechanisms: thermal — stars: neutron — stars (individual): RX J1856.5-3754 — X-rays: stars

\section{INTRODUCTION}

Despite isolated neutron stars (INSs) are mostly detected as radiopulsars (PSRs), the existence of different manifestations of INSs, with vastly diverse observational properties, emerged over ' the last two decades. These include the soft $\gamma$-repeaters and the ' anomalous X-ray pulsars (SGRs and AXPs, aka the magnetar candidates; see e.g. Mereghetti 2008; Turolla et al. 2015, for reviews), the central compact objects in supernova remnants (CCOs; ' e.g. de Luca 2008; Ho 2013), the thermally emitting X-ray INSs (also called the Magnificent seven, M7 hereafter; e.g. Turolla 2009) and the rotating radio transients (RRaTs; e.g. Burke-Spolaor 2013). The number of known objects in each class is fairly limited $(\approx 10-100)$ when compared to that of PSRs $(\approx 2000$ in the ATNF catalogue, Manchester et al. 2005 11. Still, estimates of the birth rates indicate that some INS groups may outnumber PSRs (the thermally emitting X-ray INSs and the RRaTs in particular, see Popov, Turolla \& Possenti 2006; van Kerkwijk \& Kaplan 2008) and the total INS birth rate turns out to exceed the commonly accepted value of the Galactic supernova rate Keane \& Kramer 2008). This has been taken as evidence that some evolutionary links among the INS classes must exist and led to the quest for the socalled "grand unification" of neutron stars, or GUNS for short (e.g. Kaspi 2010; Igoshev et al. 2014).

The basic idea behind GUNS is that the observational status of an INS at a given age depends on its properties at birth, - chiefly the mass, the initial spin period, and the magnetic field, - but not

\footnotetext{
* E-mail:sergepolar@gmail.com

1 The ATNF Pulsar Database, www.atnf.csiro.au/people/pulsar/psrcat/.
}

in a simple manner. The successive thermal, rotational and magnetic evolution is responsible for the outward appearance of the star, which can pass through different stages, crossing from one class to another. Thermal and magnetic evolution are inherently coupled, since the magnetic diffusivity, which enters the induction equation for the B-field, strongly depends on temperature (and density).

Models of magneto-thermal evolution of NSs provide predictions which can be tested using observational data. In particular, since the crustal magnetic field dictates the surface temperature distribution and hence the properties of the thermal emission, the observed spectra of thermally emitting INSs can be used to probe the topology of the magnetic field and its evolution. In this paper we confront the predictions of the Hall attractor model Gourgouliatos et al. 2013; Gourgouliatos \& Cumming 2014a) with X-ray spectral data of the M7. In section 2 we briefly summarize the properties of the Hall evolution and of the Hall attractor, while in section 3 we discuss which classes of INSs are best suited to this kind of investigation. Our model for the surface emission is presented in section 4 and results in section 5 Discussion follows in section 6

\section{MAGNETIC FIELD EVOLUTION AND THE HALL ATTRACTOR}

In recent years, magnetic field evolution in INSs has been the focus of several studies (see a review in Geppert 2009). As noticed by Pons \& Geppert (2007), the internal magnetic field of INSs is likely supported by two current systems, a short-lived one in the crust, with typical decay time $\tau_{\text {decay }} \approx 0.1-10 \mathrm{Myr}$ and a long- 
lasting one in the core, $\tau_{\text {decay }} \gtrsim 10-100$ Myr (see Elfritz et al. 2016, for recent calculations corroborating this picture). This implies that in not-too-old INSs, with age $\lesssim$ a few Myrs, the evolution of the core field can be ignored. This applies in particular to the M7, which have a dynamical age $\sim 0.5$ Myrs (e.g. Mignani et al. 2013, and references therein). Observationally, the picture of field decay is, however, far from being clear. The properties of the radio-pulsar population can be reproduced without invoking any decay of the magnetic field, or assuming a timescale so long that it exceeds the lifetime of a normal radio pulsar (e.g. Regimbau \& de Freitas Pacheco 2001; Faucher-Giguére \& Kaspi 2006). On the other hand, some studies have shown that synthetic populations match the observed ones if the field decays on a timescale of a few Myrs (Gonthier et al. 2002). While these calculations assumed a constant field decay rate, more recent population synthesis simulations rely on a more accurate, self-consistent modelling of the field, spin, and thermal evolution, providing results in correspondence with data (Popov et al. 2010; Gullón et al. 2014). Finally, Igoshev \& Popov (2014) demonstrated that a brief episode of relatively rapid field decay (characteristic timescale few $\times 10^{5} \mathrm{yrs}$ ) can fit well data on young and middle age radio pulsars.

We mention also that, aside from the evolution of the field itself, the rotational properties of an INS are influenced by possible variations in time of the direction of the dipole moment with respect to the star spin axis (which is measured by the angle $\xi$, see Section 4. This issue has been extensively discussed in the literature, partly in connection with magneto-rotational evolution (see Philippov et al. 2014 and references therein), partly in connection with the precession of INSs (e.g. Link 2003, for a review). In particular, it has been shown that the star tends to a global minimum energy configuration which is characterized by $\xi=0$ or $\xi=\pi / 2$ (i.e. either an aligned or an orthogonal rotator; e.g. Wasserman 2003, and references therein). While local minimum energy states (in which the INS precesses) can be achieved over relatively short timescales, the global equilibrium (when no precession occurs) requires much longer, as compared to the spin-down time. We note that the spin-down of an INS (and its thermal history) can be influenced by other effects, besides magneto-rotational losses. They include gravitational wave emission, the interaction with a fossil disc and possibly the interaction of the rotating dipole with the magnetized vacuum around the star (the so-called quantum vacuum friction; e.g. Davies 2005); Xiong. Gao \& Xu 2016). These are not addressed in the present investigation.

Here we focus on the evolution of the crustal component of the field and assume that the core field does not change in time. Moreover, given that no precession is observed in $\mathrm{RX} \mathrm{J1856} 2$, we do not consider any evolution of $\xi$. Magnetic field evolution in a INS crust proceeds mainly under the influence of two processes: Ohmic dissipation and Hall cascade (see, for example, Cumming et al. 2004). Ohmic dissipation can be due to electron scattering on phonons while the temperature is high enough. The characteristic timescale for the field evolution at this stage is $\sim 1 \mathrm{Myr}$, or even shorter depending on the magnetic field. After the temperature drops below a critical value dissipation is due to impurities, and the timescale is significantly longer. Note that these timescales are estimated for the large scale magnetic field. The Ohmic timescale is (Cumming et al. 2004):

\footnotetext{
2 Precession is indeed measurable in the M7, and it was suggested to occur
} in RX J0720.4-3125 (Hohle et al. 2012, and references therein).

$$
\tau_{\mathrm{Ohm}}=\frac{4 \pi \sigma d^{2}}{c^{2}}=4.4 \times 10^{6}\left(\frac{\sigma}{10^{20} \mathrm{~s}^{-1}}\right)\left(\frac{d}{1 \mathrm{~km}}\right)^{2} \mathrm{yr}
$$

where $\sigma$ is conductivity, $d$ the typical lengthscale of the field structure, and $c$ the speed of light. Since $d$ becomes smaller for higherorder multipolar components, small scale fields dissipate much faster.

The Hall cascade is a non-dissipative process (Goldreich \& Reisenegger 1992). It just redistributes magnetic energy, mainly transferring it from the larger to the smaller scales, where it is rapidly dissipated due to Ohmic losses. The typical timescale of the Hall process can be estimated as Cumming et al. 2004):

$$
\begin{aligned}
\tau_{\text {Hall }} & =\frac{4 \pi n_{\mathrm{e}} e d^{2}}{c B} \\
& =6.4 \times 10^{5}\left(\frac{n_{\mathrm{e}}}{10^{36} \mathrm{~cm}^{-3}}\right)\left(\frac{d}{1 \mathrm{~km}}\right)^{2}\left(\frac{B}{10^{14} \mathrm{G}}\right)^{-1} \mathrm{yr},
\end{aligned}
$$

where $n_{\mathrm{e}}$ is the electron density, and $d$ is again the typical lengthscale for $B$ (and so for currents and $n_{\mathrm{e}}$ ) in the crust. Note, that $n_{\mathrm{e}}$ and $d$ can vary, and for NSs with a large magnetic field $t_{\text {Hall }}$ can be small. So, it is expected that highly magnetized NSs undergo rapid field evolution. This is now believed to be the case for the magnetar candidates, in which rapid field dissipation is driven by field reconfiguration due to Hall cascade (Viganò et al. 2013).

Calculations by Gourgouliatos \& Cumming (2014a b), under the assumption of axial symmetry, have shown that Hall evolution saturates after a few $t_{\text {Hall }}$. The magnetic field reaches some stable configuration, and the successive evolution is driven mainly by the relatively slow Ohmic dissipation. The stage when the Hall cascade stops is called by these authors the Hall attractor and it is reached in $\lesssim 1$ Myr for magnetar-like initial fields. These picture was confirmed by the 3D numerical simulations by Wood \& Hollerbach (2015). Due to the Hall cascade the field in the crust is moved towards the crust-core boundary, and dissipates there (Gourgouliatos \& Cumming 2014b). Thus, field looks more like a core-centered field. According to Gourgouliatos \& Cumming (2014b) the Hall attractor has a well-defined property. When the field structure is stabilized, its poloidal part mainly consists of dipole and octupole components (with small addition of the $l=5$ multipole).

Since the surface thermal distribution in a cooling, magnetized INS is determined by the structure of its crustal field (e.g. Greenstein \& Hartke 1983; Page 1995; Geppert et al. 2004, 2006, see Section 4 the analysis of INS X-ray spectral properties can provide a direct test for the Hall attractor scenario.

\section{POPULATIONS OF ISOLATED NEUTRON STARS}

In order to compare with observations the predictions on the field evolution by Gourgouliatos \& Cumming, we need to select a sample of INSs which are allegedly close to the Hall attractor stage. In particular, these sources need to be of the right age $(\lesssim 1 \mathrm{Myr})$, possess initially high fields, and should display thermal emission from their cooling surface (better, not polluted by significant non-thermal magnetospheric contribution). The magneto-rotational evolution of an initially highly magnetized INSs can be approximately followed 
assuming that during the Hall stage the magnetic field decays exponentially with a characteristic time $\tau=10^{4}\left(B / 10^{15} \mathrm{G}\right)^{-1} \mathrm{yr}$ (see, for example, Aguilera et al. 2008 for discussion of simple fits for the field decay model in the presence of the Hall term). In this case the loci of constant age in the $P-\dot{P}$ plane do not coincide with the usual constant characteristic age lines. They are shown in figure 1 for an age equal to two (orange solid line) and three (light blue dashed line) Hall timescales, i.e. when the magnetic field has decayed by a factor $\exp (-2)$ and $\exp (-3)$, respectively. The asterisks along the two lines give the true age of the star in Myr (for the initial spin period equal to $0.01 \mathrm{~s}$ ). Note that an INS will reach the line at different times according to its initial magnetic field, since the Hall timescale depends on $B_{0}$.

According to figure 1, while some old radio pulsars can indeed reach the Hall attractor, on average objects of this type have too low magnetic fields, and hence too long Hall timescales, in agreement with the results by Igoshev \& Popov (2015). Active magnetars appear also to be far from the attractor stage, as expected, since after the attractor is reached the release of magnetic energy, and hence the activity, should decrease (see, however, Wood \& Hollerbach 2015, who claim that old magnetars can still produce bursts, even after reaching the attractor stage).

Quite interestingly, figure 1 shows that close-by, cooling isolated NSs, the XDINSs or M7, lie close to the Hall attractor stage, as quite naturally follows if these sources are descendants of magnetars (so that their initial field was huge), with typical ages $\sim 0.5 \mathrm{Myr}$ (Popov et al. 2010). This is very fortunate, as the M7 are purely thermal sources, and their surface emission is potentially sensitive to the magnetic field structure in the crust. Indirectly, the proximity of the M7 to the attractor may be also supported by their low activity. In the next subsection we briefly summarize the properties of X-ray emission from the M7.

\subsection{X-ray emission from the Magnificent Seven}

The M7 are isolated neutron stars characterized by stable thermal emission with temperatures $\sim 50-100 \mathrm{eV}^{3}$. Typically, their spectra can be fitted by one or two blackbody components with the addition of a broad absorption feature at few hundred eVs. The pulsed fraction is usually low $(\lesssim 20 \%)$ and the luminosities are in the range $L \sim 10^{31}-10^{32} \mathrm{erg} \mathrm{s}^{-1}$. A recent summary of the main properties of the M7 can be found in Pires et al. (2014, see also Turolla 2009).

The spin-down measure of the dipole field in the M7 gives values somehow in excess of those typical of radio pulsars and not too far from those of the magnetars, i.e. $10^{13}-10^{14} \mathrm{G}$ (e.g. Turolla 2009 This led to the suggestion that the M7 could be elderly magnetars, kept hotter than normal INSs of the comparable age by field decay (Pons et al. 2007). Indeed, detailed population synthesis calculations confirmed that the general properties of the M7 follow naturally in the framework of a decaying magnetic field (Popov et al. 2010).

Different sources among the M7 can be at different evolutionary stages, and so they can be closer or farther from the attractor stage. This may translate into different levels of activity, since, according to Gourgouliatos \& Cumming (2014b) sources close to the

${ }^{3}$ See the on-line catalogue of thermally emitting NSs at http://neutronstarcooling.info Viganò et al. 2013).

4 These values are in agreement with those obtained from the energy of the absorption features, assuming that the latter are produced by proton cyclotron resonance or by bound-bound transitions in low-Z elements.

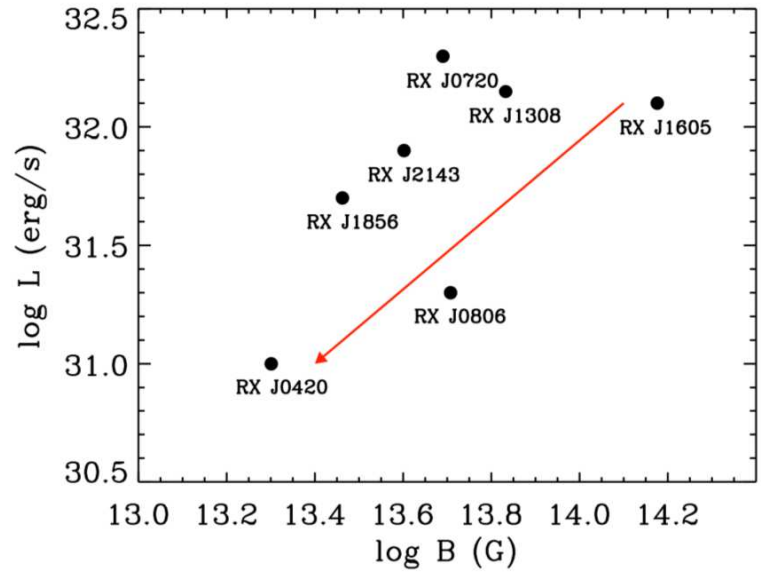

Figure 2. The placement of the $\mathrm{M} 7$ in $B-L$ plane (data taken from http://www.neutronstarcooling.info//). The red arrow indicates the likely evolutionary path of a NS during the Hall phase.

attractor should appear less active. Actually, none of the M7 exhibits magnetar-like activity. Still, they display somehow different characteristics, and this can be used to gauge the proximity to the attractor (see Fig. 2]. In particular, NSs at (or very near) the attractor should have lower pulsed fraction and lower magnetic field than other sources of the same class, together with lower temperatures and luminosities, since it is expected that, in the case of the M7, temperatures and luminosities are slightly enhanced due to field decay (Popov et al. 2010). Following this line, RX J0720.4-3125 may provide a good case for an M7 source which is still far from the Hall attractor. In fact, it has the largest luminosity among the Seven, its pulsed fraction is $>10 \%$, and it exhibits long-term spectral variability (e.g. Van Kerkwick et al. 2007; Hohle et al. 2012, and references therein). On the other hand, RX J0420.0-5022 and RX J1856.5-3754 may be representatives of sources close to the Hall equilibrium: they have low blackbody temperatures $(\sim 50-60$ $\mathrm{eV})$, and hence luminosities, and their dipolar B-field, $\sim 10^{13} \mathrm{G}$, is the weakest among the Seven.

At variance with RX J0420.0-5022, RX J1856.5-3754 is the prototype and brighter member of the class, and its spectral properties are very well-characterized. The pulsed fraction is the lowest, $\sim 1 \%$, no variability was detected so far and the X-ray spectrum is well fitted by two blackbody components with $k T_{1}^{\infty} \sim 61-$ $62 \mathrm{eV}, R_{1}^{\infty} \sim 4.5-5 \mathrm{~km}$ and $k T_{2}^{\infty} \sim 39 \mathrm{eV}, R_{2}^{\infty} \sim 11-16 \mathrm{~km}$ (Sartore et al. 2012); all the previous quantities are referred to an observer at infinity. For these reasons in the following we focus on RX J1856.5-3754 (RX J1856 hereafter), and try to assess if its spectral properties are indeed compatible with the crustal distribution of the magnetic field predicted by the Hall attractor configuration.

\section{THE MODEL}

In order to compute synthetic spectra from the cooling surface of an isolated NS we follow the same approach presented in Zane \& Turolla (2006, see also Page 1995; (Taverna et al. 2015). The star surface is divided in a number of patches by means of an equally-spaced grid in $\mu \equiv \cos \Theta$ and $\Phi(0 \leqslant \mu \leqslant 1$, $0 \leqslant \Phi \leqslant 2 \pi$ ), where $\Theta$ and $\Phi$ are the co-latitude and the az- 


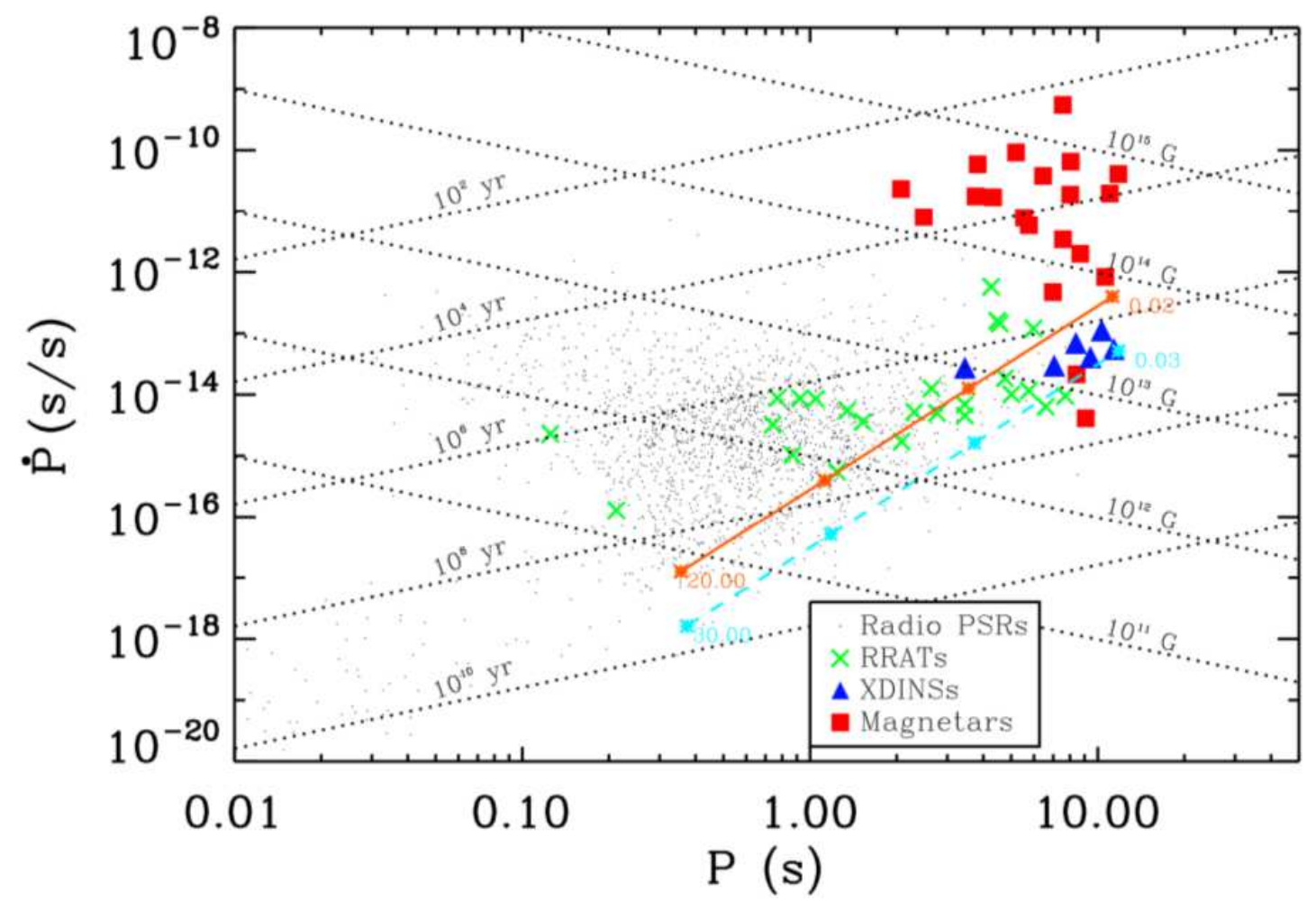

Figure 1. The $P-\dot{P}$ diagram showing the different INS classes. The full orange (dashed light blue) line corresponds to an age equal to two (three) Hall timescales (see text); here an initial period $P_{0}=0.01 \mathrm{~s}$ has been assumed and the initial magnetic field is in the range $10^{12} \mathrm{G} \leqslant B_{0} \leqslant 10^{15} \mathrm{G}$. The asterisks along the two lines mark the true age of the star in Myrs, the spacing between two symbols corresponds to a factor of 10 decrease, moving from left to right.

imuth relative to the line-of-sight (LOS). Relativistic ray-bending (the vacuum Schwarzschild solution is assumed throughout) is accounted for by introducing the angle $\bar{\Theta}$,

$$
\bar{\Theta}=\int_{0}^{1 / 2} \frac{d v \sin \Theta}{\left[(1-x) / 4-(1-2 v x) v^{2} \sin \Theta^{2}\right]^{1 / 2}},
$$

so that the monochromatic flux emitted by the portion of the surface which is into view at a given spin phase $\gamma$ is given by

$$
F_{\nu}(\gamma)=(1-x) \frac{R_{\mathrm{NS}}^{2}}{D^{2}} \int_{0}^{2 \pi} d \Phi \int_{0}^{1} I_{\nu}(\mathbf{k}, \theta, \phi) d u^{2}
$$

here $u=\sin \bar{\Theta}, D$ is the source distance, $x=R_{\mathrm{s}} / R_{\mathrm{NS}}, R_{\mathrm{s}}=$ $2 G M_{\mathrm{NS}} / c^{2}$ and $M_{\mathrm{NS}}, R_{\mathrm{NS}}$ are the star mass and radius. In equation (4), $I_{\nu}$ is the specific intensity which in general depends on the photon direction $\mathbf{k}$, and on the position of the emitting point on the surface, expressed here through the magnetic co-latitude $\theta$, and azimuth $\phi$. The components of $\mathbf{k}, \theta$ and $\phi$ can, in turn, be expressed in terms of $\Theta, \Phi, \gamma$ and the two geometrical angles $\chi$ and $\xi$ which give, respectively, the inclination of the LOS and of the magnetic axis with respect to the star spin axis.

In the following we restrict to magnetic configurations described by the superposition of multipoles up to $l=5$ (see section 2]; we specify the strength of the dipole at the magnetic pole, $B_{\mathrm{p}}$, and the polar ratio of the $l$-multipole to the dipole, $\rho_{1}$. Generalrelativistic corrections to the $B$-field were included through the functions $f(x)$ and $g(x)$, for the $r$ - and $\theta$-component of the field, respectively (Muslimov \& Tsygan 1986. For magnetic fields $\gtrsim$ $10^{11} \mathrm{G}$ electron thermal conduction is essentially along the magnetic field lines and the surface temperature distribution is given by

$$
T_{\mathrm{s}} \simeq T_{\mathrm{p}}\left|\cos \theta_{\mathrm{B}}\right|^{1 / 2},
$$

where $T_{\mathrm{p}}$ is the polar value of the temperature and $\theta_{\mathrm{B}}$ is the angle between B and the surface normal (e.g. Greenstein \& Hartke 1983; Page 1995, see also Potekhin, Pons \& Page 2015).

No definite physical model for the surface emission from the M7 has been put forward as yet (see e.g. Potekhin 2014, for a discussion). It has been suggested that in these sources the surface layers are in a condensed state, owing to a phase transition driven by the low surface temperature and relatively high magnetic field (Lai \& Salpeter 1997; Burwitz et al. 2003; Turolla, Zane \& Drake 2004; Medin \& Lai 2007, see also Turolla 2009, Potekhin 2014). In particular, Ho et al. (2007) used the condensed surface emission model to explain the multiwavelength spectral energy distribution

\footnotetext{
5 Note that for higher order multipoles, the analytical expression for the hypergeometric functions, which give $f$ and $g$, becomes numerically unstable even for $x \sim 0.3$. For this reason we used the sum of the (truncated) hypergeometric series instead.
} 
of RX J1856, with the addition of a thin, magnetized, $\mathrm{H}$ atmosphere on top of the condensate to reproduce the optical spectrum. In the next section we present results for the X-ray spectrum of RX J1856 for magnetic configurations typical of the Hall attractor for both (isotropic) blackbody emission at the local temperature $T$ and a condensed surface. The specific intensity in the former case is simply

$$
I_{\nu, \mathrm{BB}}=B_{\nu}(T)=\frac{2 h}{c^{2}} \frac{\nu^{3}}{\exp (h \nu / k T)-1}
$$

while in the latter it can be expressed as

$$
I_{\nu, \mathrm{C}}=j_{\nu}\left(B, \mathbf{k}, \theta_{\mathrm{Bk}}\right) B_{\nu}(T),
$$

where $j_{\nu}$ is the emissivity of the condensed phase and $\theta_{\mathrm{Bk}}$ is the angle between the photon direction and the magnetic field; the analytical approximations by Potekhin et al. (2012) were used to compute $j_{\nu}$ (see also González Caniulef et al.2016, for more details). Because of the present uncertainties in modelling the dielectric tensor of the condensed phase, here we consider both the two limiting cases in which ions are either treated as "free" or "fixed" (see e.g. Turolla, Zane \& Drake 2004; Potekhin et al. 2012, for more details).

\section{NUMERICAL RESULTS}

The spectral properties of RX J1856 were simulated assuming a NS with mass $M_{\mathrm{NS}}=1.4 \mathrm{M}_{\odot}$ and radius $R_{\mathrm{NS}}=12 \mathrm{~km}$; we also assumed a polar temperature at the surface $T_{\mathrm{p}} \simeq 75 \mathrm{eV}$, compatible with the estimated temperature of the hotter blackbody at infinity by (Sartore et al. 2012, see 3.16 . The temperature profile given by equation (5) was slightly modified, by truncating it at $T_{\mathrm{e}}=5 \mathrm{eV}$ in order to avoid a vanishing temperature at the magnetic equator (see e.g. Taverna et al. 2015; González Caniulef et al. 2016). Following Gourgouliatos \& Cumming (2014b), we mimicked the Hall attractor stage adopting two different magnetic field topologies: a combination of dipolar and octupolar components, with opposite polarity and polar ratio $\rho_{3}=0.6$, and a similar configuration, with the addition of an $\ell=5$ component, characterized by the polar ratios $\rho_{3}=0.6$ and $\rho_{5}=0.3$. Hereafter we will refer to these two models as model 1 and model 2, respectively. According to the measured values of $P$ and $\dot{P}$, we set the magnetic field intensity for the dipolar component at the poles at $B_{\mathrm{p}} \simeq 10^{13} \mathrm{G}$ van Kerkwijk \& Kaplan 2008).

The resulting maps for the surface temperature are shown in Fig. 3b for model 1 and Fig. 3r for model 2; the map for a purely dipolar magnetic field is also shown for comparison. Model 1 and 2 only differ for a small region around the magnetic pole: here the surface temperature is $\sim 25 \%$ lower with respect to $T_{\mathrm{p}}$ for model 1 , while, at the same magnetic colatitudes, it is nearly constant (and equal to the polar value) for model 2 . On the other hand, comparing the first two maps with that for the purely dipolar field, it turns out that the addition of higher-order multipoles generally increases the surface temperature, making its distribution more uniform. This implies that the pulsed fraction will likely decrease as the star approaches the Hall attractor.

We then simulated the emission, first considering the case of surface blackbody radiation with temperature $T=\max \left(T_{\mathrm{s}}, T_{\mathrm{e}}\right)$

6 The gravitational redshift factor for the values of mass and radius used here is $1+z \sim 1.24$.
Surface field structure in RX $J 1856$

\begin{tabular}{lccccc}
\hline & $\chi$ & $\xi$ & $T_{1}(\mathrm{eV})$ & $T_{2}(\mathrm{eV})$ & $A_{2} / A_{1}$ \\
\hline Pure dipole & $15^{\circ}$ & $80^{\circ}$ & 72.0 & 57.8 & 1.27 \\
\hline Model 1 & $20^{\circ}$ & $80^{\circ}$ & 73.0 & 59.4 & 0.76 \\
\hline Model 2 & $25^{\circ}$ & $80^{\circ}$ & 73.5 & 58.1 & 0.36 \\
\hline
\end{tabular}

Table 1. Results of the fits performed for the spectra shown in Figure 5 The values of temperature are at the star surface.

(see equation 5. The top row of Figure 4 shows the pulsed fractions, in the $0.1-1.6 \mathrm{keV}$ energy range, for the pure dipole (left), model 1 (center) and model 2 (right), plotted as function of the angles $\chi$ and $\xi$. The decrease of the pulsed fraction as higher-order multipoles are added to the dipole is apparent from Figure 4 The loci in the $(\chi, \xi)$ plane that correspond to the observed value of the pulsed fraction for RX J1856 ( 1.3\%, see 3.1), are also drawn to show the geometrical configurations which correspond to the observational data.

In order to check if the spectral parameters derived by Sartore et al. (2012) are indeed reproduced, we calculated the phase-averaged spectra in the $0.1-2 \mathrm{keV}$ energy range for each pair of angles $\chi$ and $\xi$ and fitted them with two blackbody components at temperature $T_{1}$ and $T_{2}$

$$
F=A_{1} E^{3}\left(\frac{1}{\exp \left(E / k T_{1}\right)-1}+\frac{A_{2} / A_{1}}{\exp \left(E / k T_{2}\right)-1}\right),
$$

where the normalization $A_{1}\left(A_{2}\right)$ depends only on the emitting area. The middle and bottom rows of Figure 4 show, again as a function of $\chi$ and $\xi$, the behavior of the ratios $T_{2} / T_{1}$ and $A_{2} / A_{1}$, respectively, for the same three magnetic configurations described above. Moving from the dipole towards the Hall attractor stage, the ratio $T_{2} / T_{1}$ increases slightly, being anyway close to $\sim 0.7$, not that far from the value $(\sim 0.65)$ derived by Sartore et al. (2012). On the contrary, the ratio $A_{2} / A_{1}$ generally decreases. In particular, only in the case of the dipole, and for some viewing geometries (small $\chi$ - large $\xi$ and vice versa), the emitting area of the colder component is larger than the hotter one, with $A_{2} \sim 1.2 A_{1}$. Adding higher-order multipole components, instead, $A_{2}$ becomes systematically smaller than $A_{1}$, resulting in $A_{2} \sim 2 / 3 A_{1}$ for model 1 and $A_{2} \sim 1 / 3 A_{1}$ for model 2 . This behavior contrasts with the observations, which give an emitting area ratio in the range $\sim 6-10$.

As an example, Figure 5 shows the spectra, as observed at infinity, for the dipole (top), model 1 (center) and model 2 (bottom), for values of the angles $\chi$ and $\xi$ chosen in such a way that the pulsed fraction is compatible with the observed value (see Figure 47. The individual blackbody components used to fit the spectra are also shown; the fit results are summarized in Table 1 As discussed above, it is clear that there is no way to reproduce the observed emitting area ratio $A_{2} / A_{1}$ with the magnetic field topologies we used to approximate the Hall attractor stage, although in all the three cases spectra are well fitted with two blackbody curves with temperatures compatible with those derived by Sartore et al. (2012).

Finally, we performed the same simulations assuming that emission is from a condensed surface, using the analytical approximations by Potekhin et al. (2012), both in the free-ions and fixedions limits. We found that, for all the magnetic field configurations considered, a fit with two blackbody components (as given by equation 8 fails match the observations, with a ratio $A_{2} / A_{1}$ smaller than $1 \%$. In particular, Figure 6 clearly shows that, for the case of model 1 , the shape of the spectra obtained for the condensed sur- 


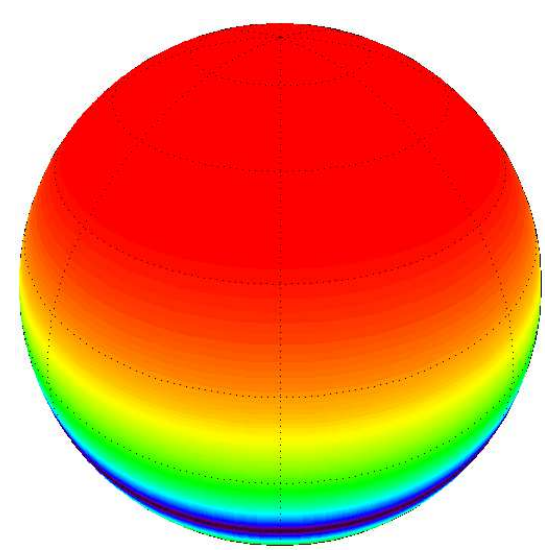

(a)

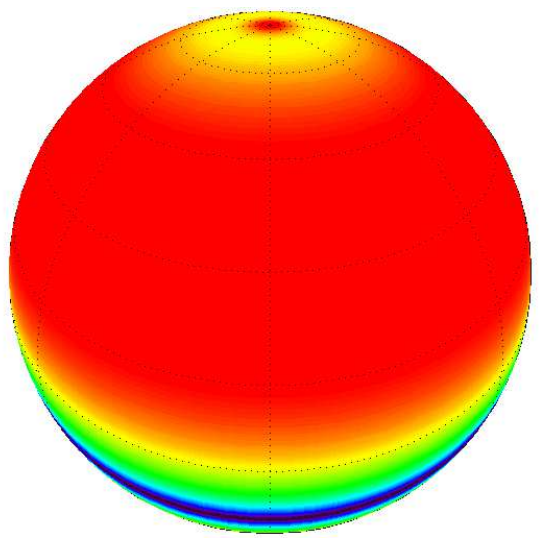

(b)

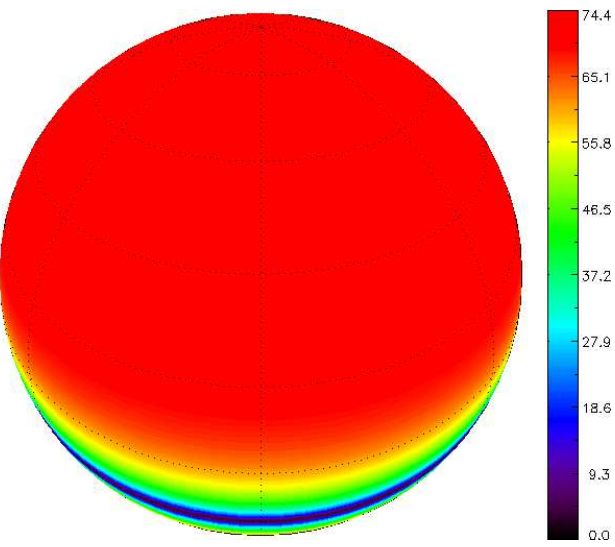

(c)

Figure 3. Temperature maps for the three different magnetic field topologies discussed in the text: pure dipole (left), dipole plus octupole (model 1, center) and dipole plus octupole plus $\ell=5$ component (model 2, right).

face emission model (middle and bottom panels) requires the presence of at least another component in order to explain the spectral hardening that is present at energies around $0.4-0.5 \mathrm{keV}$, at variance with what happens for the isotropic blackbody emission (top panel), where the spectral enhancement is not present. Similar results have been found in the cases of model 2 and purely dipolar field.

In summary, our results indicate that for the field configuration predicted by the Hall attractor, the area corresponding to the hotter temperature is larger than that at the cooler temperature, contrary to what is observed in RX J1856.

\section{DISCUSSION}

It has been recently suggested that the magnetic field evolution in isolated neutron stars proceeds through a phase dominated by the Hall cascade until a Hall equilibrium stage (the Hall attractor) is reached (Gourgouliatos \& Cumming 2014a b; Wood \& Hollerbach 2015). The Hall attractor is characterized by a well defined structure of the crustal magnetic field and, in turns, of the surface temperature distribution. The latter can, in principle, be probed through X-ray observations of thermally emitting INSs, and, in this respect, the "Magnificent Seven" (M7) provide an optimal target. In this paper we have compared the predictions of the Hall attractor scenario with the observed spectral properties of the brightest of the M7, RX J1856.5-3754. Our main conclusion is that the surface temperature distribution produced by the Hall attractor magnetic field configuration fails to explain the X-ray spectrum of RX J1856.5-3754, as derived from XMM-Newton observations by Sartore et al. (2012). In particular, while the temperatures of the two blackbody components which best-fit the data are broadly reproduced, the emitting areas are not.

The nature of the surface emission from cooling neutron stars is still not completely understood. For this reason we considered both isotropic blackbody emission and emission from a condensed phase, both at the local temperature. Agreement with observations was not found in either case, irrespectively of the geometry of the source (inclination of the line-of-sight and of the magnetic axis with respect to the rotation axis). Although it can not be excluded that other emission models can provide a better agreement with data, we deem this unlikely.

General-relativistic ray-bending was properly accounted for in our calculations. Numerical results were obtained for reasonable values of the star mass and radius, $1.4 M_{\odot}$ and $12 \mathrm{~km}$, respectively, and hence for the $M / R$ ratio. However, since no estimate for these two quantities is available for RX J1856.5-3754, one may wonder if other choices for $M_{\mathrm{NS}}$ and $R_{\mathrm{NS}}$ could change our conclusions. While we did not attempt a systematic exploration, results obtained for other values, e.g. $M_{\mathrm{NS}}=1.2 M_{\odot}$ and $R_{\mathrm{NS}}=15 \mathrm{~km}$, are in qualitative agreement with previous ones, with only some marginal quantitative differences.

\subsection{Comparison between RX J1856 and RX J0720}

It is interesting to compare properties of the two most studied sources among the M7: RX J1856 and RX J0720. In particular, which one is older, and what can we say about their initial parameters and evolution.

For both objects age determinations are not very precise. The characteristic age, $\tau_{\mathrm{ch}}=P / 2 \dot{P}$, is slightly larger for RX J1856: $\log \tau_{\text {ch }}=6.58$ vs. 6.28 (Pires et al. 2014). But the kinematic age is larger for RX J0720: $\log \tau_{\text {kin }}=5.93$ vs. 5.62 (Pires et al. 2014). Note, that RX J1856 has slightly smaller spin period and smaller $\dot{P}$ (and so, smaller magnetic field $B \propto \sqrt{P \dot{P}}$ ).

In our considerations related to the magneto-rotational evolution we noted that RX J1856 is less active (it has smaller pulse fraction, luminosity, and temperature, it does not demonstrate strong variability, etc.). In Figs. 1 2 RX J1856 is situated closer to the Hall attractor.

Taking all together, we have to conclude that RX J1856 is younger (smaller $\tau_{\text {kin }}$ ), but more evolved. It is possible if its magnetic field was initally larger, and then the source evolved faster. However, in standard (simplified) models of evolution with field decay, if two NSs have similar (small) initial periods, but significantly different magnetic fields, then the less magnetized cannot attain longer spin period and, at the same time, higher $\dot{P}$. Then, some complications to explain the data on RX J1856 and RX J0720 are necessary. To give a visual impression, we can say that evolutionary tracks of RX J1856 and RX J0720 might cross on the 

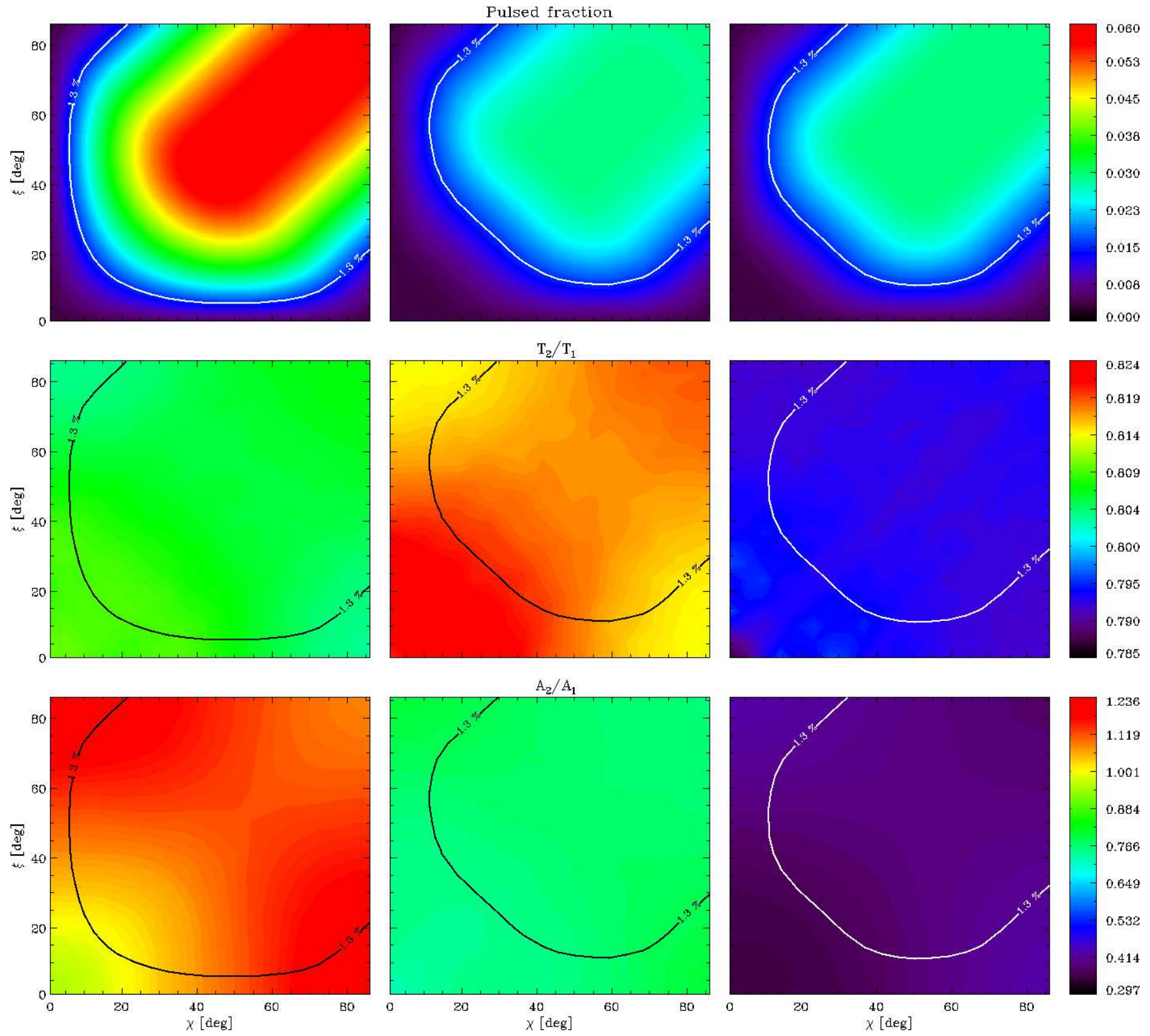

Figure 4. Contour plots for the pulsed fraction (top row), the ratios of the temperatures (middle row) and emitting areas (bottom row) of the two blackbody components which fit the phase-averaged spectra, plotted as functions of the viewing angles $\chi$ and $\xi$ for a pure dipole (left column), model 1 (middle column) and model 2 (right column); here the pulsed fraction is computed in the 0.1-1.6 keV energy range and local emission is blackbody. The curve corresponding to the observed pulsed fraction for RX J1856 ( 1.3\%) is also shown.

$P-\dot{P}$ diagram. Potentially, this can be related to some non-trivial field evolution, so that RX J1856 experienced a period of very rapid field decay in its past.

\subsection{How to find INSs at the Hall attractor}

It seems that even RX J1856 is not at the stage of the attractor, yet (if predictions are correct). Then it is necessary to find more evolved relatives of the M7.

If the Hall cascade saturates, then the field evolution is governed only by the Ohmic processes. As a NS cools down scattering of electrons on phonons becomes less important. There is a critical value of the temperature, $T_{\mathrm{U}}$ (see, for example, Cumming et al.
2004 and references therein), below which the Ohmic dissipation is determined only by impurities. Then, the field decays very slowly, and so the crust is not additionally heated. It seems that mostly NSs at the Hall attractor might be relatively cool sources. It would be very difficult to detect them even with the eROSITA (Predehl et al. 2011), as this instrument is fitted for larger temperatures.

Still, taking into account that the solar proximity is overabundant in NSs with ages $\sim 10^{6}$ yrs due to the Gould Belt (Popov et al. 2003), we can expect that dozens of INSs with $T \sim$ a few tens of $\mathrm{eV}$ are floating in space around us.

Another option is to look for thermal emission of RRaTs and pulsars which are potentially close to the Hall attractor (see Fig. 1) 


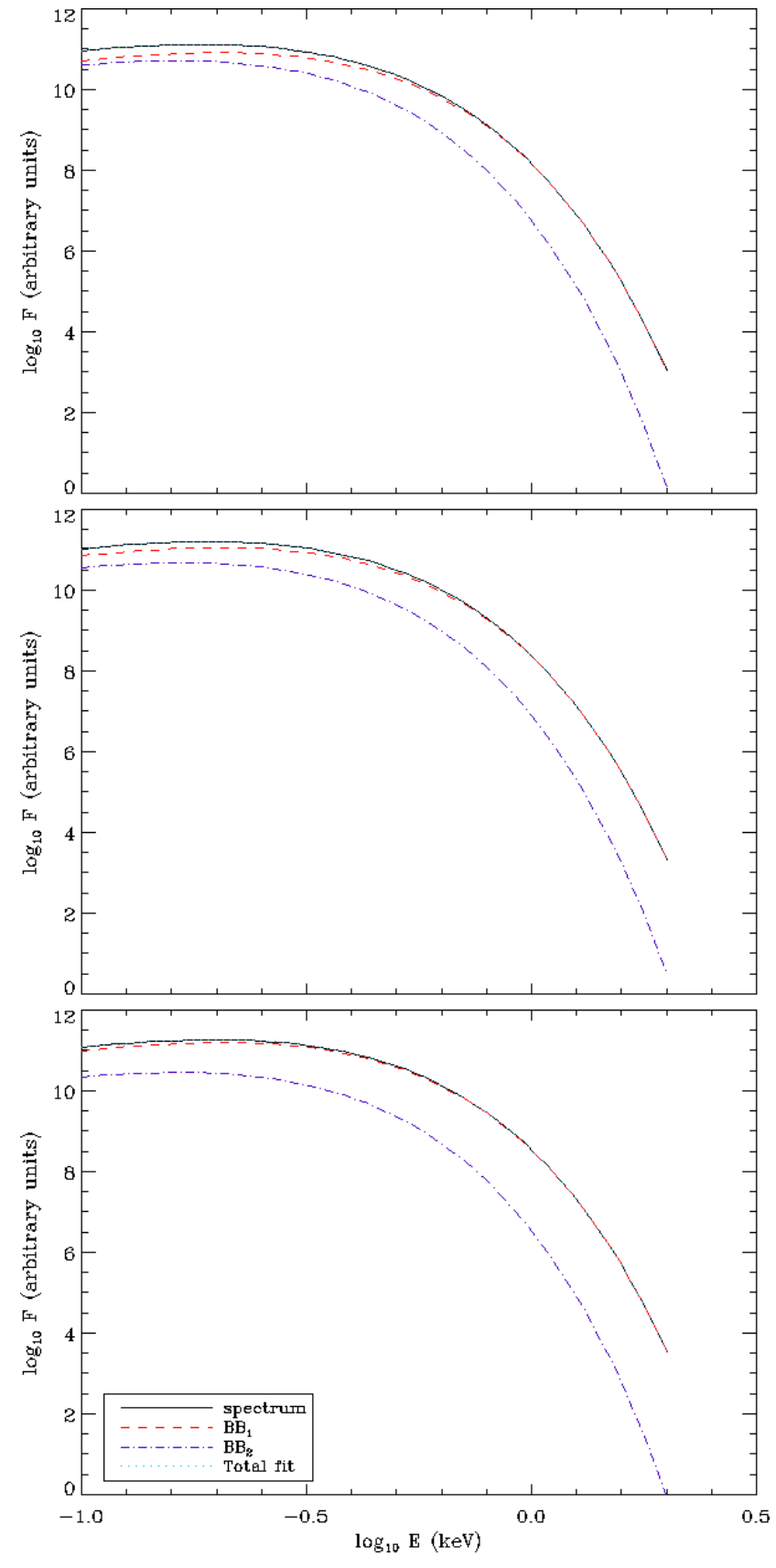

Figure 5. Phase-averaged spectra in the $0.1-1.6 \mathrm{keV}$ energy range (black solid lines) in the cases of the dipole (top panel), model 1 (middle panel) and model 2 (bottom panel), for $\xi \simeq 80^{\circ}$ and $\chi \simeq 15^{\circ}, 20^{\circ}$ and $25^{\circ}$, respectively (see Figure 4). The two blackbody components (red dashed line and blue dash-dotted line) used to fit the spectra, as well as the bestfitting curve (green dotted line) are also shown. In all the three cases local emission is an isotropic blackbody (see text for details).

and have ages $\lesssim$ Myr. For near-by sources $(\lesssim 1-2 \mathrm{kpc})$ with long exposures it is possible to detect thermal emission in X-rays.

It seems that observations of thermal emission is one of the best way to probe the existence of the Hall attractor and test its predicted properties.
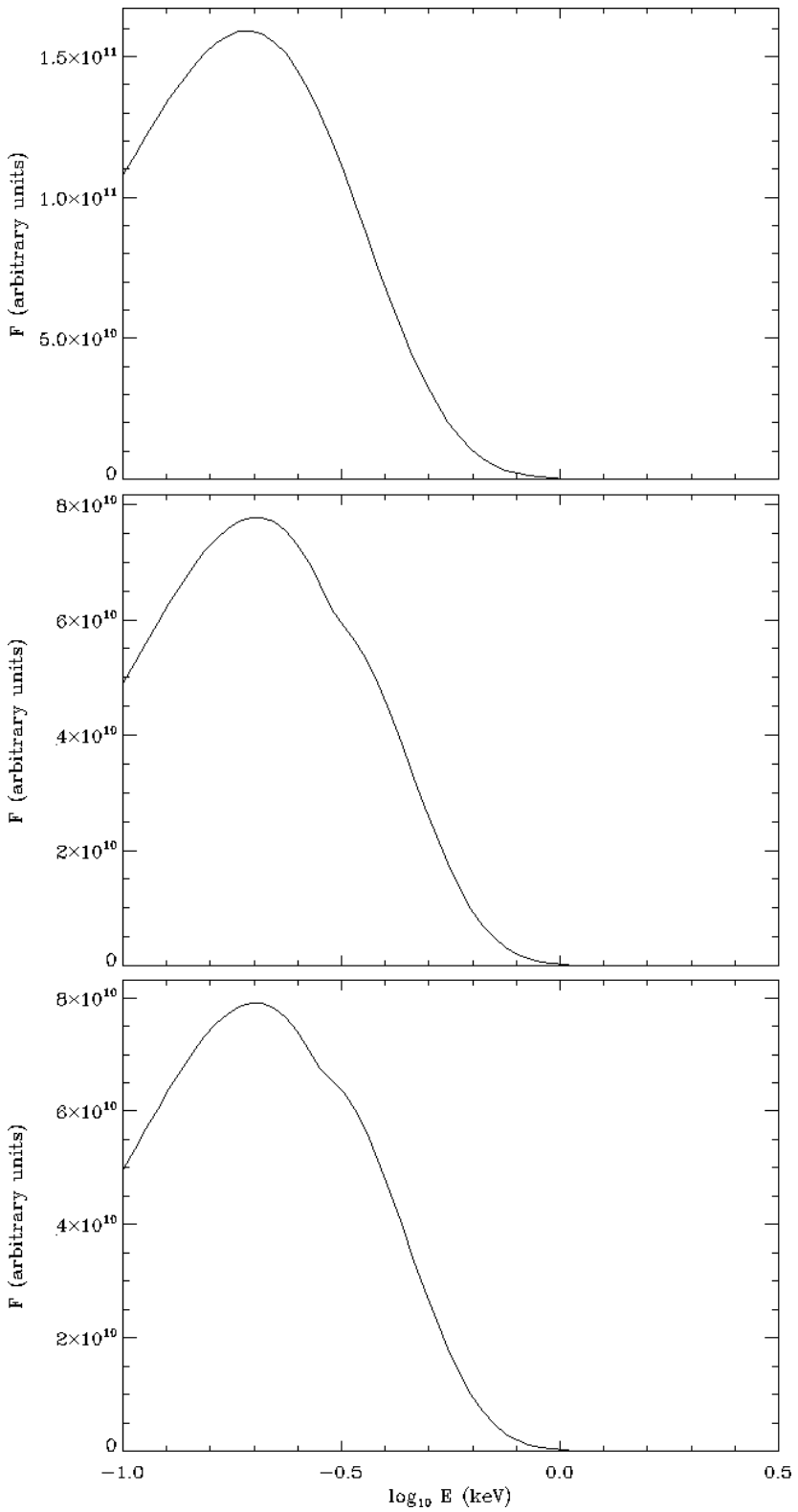

Figure 6. Phase-averaged spectra in the $0.1-1.6 \mathrm{keV}$ energy range in the case of model 1 for different surface emission models: blackbody (top panel) and condensed surface (free-ions, middle panel; fixed-ions, bottom panel). The viewing angles are chosen in such a way that the corresponding pulsed fractions are compatible with the observed value $(\sim 1.3 \%)$, i.e. $\chi \simeq 20^{\circ}, \xi \simeq 80^{\circ}$ for the blackbody and $\chi \simeq 25^{\circ}, \xi \simeq 5^{\circ}$ for the condensed surface.

\section{CONCLUSION}

In this paper we tried to verify predictions for the magnetic field evolution in NSs made by Gourgouliatos \& Cumming (2014b). Namely, we probed existence of the Hall attractor using data on surface thermal emission of the source RX J1856.5-3754. As modelling of the magnetic field structure performed by Gourgouliatos \& Cumming (2014b) predicted relative contribution of different multiipoles, we were able to calculate the expected spectral characteristics of such a NS. We found that, contrary to observations of RX J1856, at the stage of Hall attractor in the two blackbody approximation, the area corresponding to higher temper- 
ature is larger than the area related to lower temperature. Thus, we conclude that in the case of RX J1856 (and, most probaly, also in case of most or all other M7 sources) the stage of the Hall attractor is not reached, or the field structure at this stage is different from the tested predictions.

\section{ACKNOWLEDGMENTS}

SBP is grateful to the Department of Physics and Astronomy of the University of Padova for financial support and hospitality during a visit when part of this work was carried out. The work of SBP was also partially supported by the Russian Foundation for Basic Research, project 17-02-00360. SBP thanks Andrei Igoshev for several discussions. The work of RT is partially supported by INAF through a PRIN grant.

\section{REFERENCES}

Aguilera, D. N., Pons, J. A., Miralles, J. A., 2008, ApJ, 673, L167 Burke-Spolaor S., 2013, in van Leeuwen J., ed., Neutron Stars and Pulsars: Challenges and Opportunities after 80 years Vol. 291 of IAU Symposium, Rotating Radio Transients and their place among pulsars. pp 95-100

Burwitz, V., Haberl, F., Neuhäuser, R., Predehl, P., Trümper, J., Zavlin, V.E. 2003, A\&A, 399, 1109

Davies, P.C.W. 2005, Journal of Optics B, 7, 40

Cumming A., Arras P., Zweibel E., 2004, ApJ, 609, 999

de Luca A., 2008, in Bassa C., Wang Z., Cumming A., Kaspi V. M., eds, 40 Years of Pulsars: Millisecond Pulsars, Magnetars and More Vol. 983 of American Institute of Physics Conference Series, Central Compact Objects in Supernova Remnants. pp 311-319

Elfritz J. G., Pons J. A., Rea N., Glampedakis K., Viganò D., 2016, MNRAS, 456, 4461

Faucher-Giguére C-A., Kaspi V., 2006, ApJ, 643, 332

Geppert U., Küker M., Page D., 2004, A\&A, 426, 267

Geppert U., Küker M., Page D., 2006, A\&A, 457, 937

Geppert U., 2009, in Becker W., ed., Astrophysics and Space Science Library Vol. 357 of Astrophysics and Space Science Library, Turning Points in the Evolution of Isolated Neutron Stars'Magnetic Fields. p. 319

Goldreich P., Reisenegger A., 1992, ApJ, 395, 250

Gonthier P.L., Oullette M.S., Berrier J., O’Brien S., Harding, A.K., 2002, ApJ, 565, 482

González Caniulef D., Zane S., Taverna R., Turolla R. \& Wu K. 2016, MNRAS, 459, 3585

Gourgouliatos K. N., Cumming A., 2014a, Physical Review Letters, 112,171101

Gourgouliatos K. N., Cumming A., 2014b, MNRAS, 438, 1618

Gourgouliatos K. N., Cumming A., Reisenegger A., Armaza C., Lyutikov M., Valdivia J. A., 2013, MNRAS, 434, 2480

Greenstein, G., Hartke, G.J. 1983, ApJ, 271, 283

Gullón M., Miralles J.A., Viganó D., Pons J.A., 2014, MNRAS, 443, 1891

Ho, W.C.G., Kaplan, D.L., Chang, P., van Adelsberg, M., Potekhin, A.Y. 2007, MNRAS, 375, 821

Ho W. C. G., 2013, in van Leeuwen J., ed., Neutron Stars and Pulsars: Challenges and Opportunities after 80 years Vol. 291 of IAU Symposium, Central compact objects and their magnetic fields. pp 101-106
Hohle, M. M., Haberl, F., Vink, J., de Vries, C. P., Turolla, R., Zane, S., Méndez, M. 2012, MNRAS, 423, 1194

Igoshev A. P., Popov S. B., 2015, Astronomische Nachrichten, 336, 831

Igoshev A. P., Popov S. B., 2014, MNRAS, 444, 1066

Igoshev A. P., Popov S. B., Turolla R., 2014, Astronomische Nachrichten, 335, 262

Kaspi V. M., 2010, Proceedings of the National Academy of Science, 107, 7147

Keane, E. F., Kramer, M., 2008, MNRAS, 391, 2009

Lai, D., Salpeter, E.E. 1997, ApJ, 491, 270

Link, B. 2003, in Radio Pulsars, ASP Conference Proceedings, Vol. 302, p. 241. Edited by M. Bailes, D.J. Nice, S.E. Thorsett. San Francisco: Astronomical Society of the Pacific

Manchester, R. N., Hobbs, G. B., Teoh, A., Hobbs, M. 2005, AJ, 129, 1993

Medin, Z., Lai, D. 2007, MNRAS, 382, 1833

Mereghetti S., 2008, A\&A Rev., 15, 225

Mignani, R.P., Vande Putte, D., Cropper, M. et al. 2013, MNRAS, 429, 3517

Muslimov A.G., Tsygan A.I., 1986, Sov. Astr., 30, 567

Page D., 1995, Space Sci. Rev., 74, 437

Philippov A., Tchekhovskoy A., Li J.G., 2014, MNRAS, 441, 1879

Pires A. M., Haberl F., Zavlin V. E., Motch C., Zane S., Hohle M. M., 2014, A\&A, 563, A50

Pons J. A., Geppert U., 2007, A\&A, 470, 303

Pons J. A., Link B., Miralles J.A., Geppert U., 2007, Phys. Rev. Lett., 98, id. 071101

Popov S. B., Turolla, R., Possenti, A., 2006, MNRAS, 369, L23

Popov S. B., Colpi M., Prokhorov M. E., Treves A., Turolla R., 2003, A\&A, 406, 111

Popov S. B., Pons J. A., Miralles J. A., Boldin P. A., Posselt B., 2010, MNRAS, 401, 2675

Potekhin, A.Y., Suleimanov, V.F., van Adelsberg, M., Werner, K. 2012, A\&A, 546, A121

Potekhin, A.Y. 2014, Phys. Usp., 57, 735

Potekhin A. Y., Pons J. A., Page D. 2015, Space Sci. Rev., 191, 239

Predehl P., et al., 2011, in Society of Photo-Optical Instrumentation Engineers (SPIE) Conference Series. p. 81450D

Regimbau T., de Freitas Pacheco J.A., 2001, A\&A, 374, 182

Sartore N., Tiengo A., Mereghetti S., De Luca A., Turolla R., Haberl F., 2012, A\&A, 541, A66

Taverna R., Turolla R., González Caniulef D., Zane S., 2015, MNRAS, 454, 3254

Turolla, R., Zane, S.,\& Drake, J.J. 2004, ApJ, 603, 265

Turolla R., 2009, in Becker W., ed., Astrophysics and Space Science Library Vol. 357 of Astrophysics and Space Science Library, Isolated Neutron Stars: The Challenge of Simplicity. p. 141

Turolla R., Zane S., Watts A. L., 2015, Reports on Progress in Physics, 78, 116901

van Kerkwijk M. H., Kaplan D. L., 2008, ApJL, 673, L163

van Kerkwijk, M. H., Kaplan, D. L., Pavlov, G. G., Mori, K. 2007, ApJ, 659, L149

Viganò D., Rea N., Pons J. A., Perna R., Aguilera D. N., Miralles J. A., 2013, MNRAS, 434, 123

Wasserman, I. 2003, MNRAS, 341, 1020

Wood T. S., Hollerbach R., 2015, Physical Review Letters, 114, 191101

Xiong, X.-Y., Gao, C.-Y., Xu, R.-X. 2016, RAA, 16, 9 
Zane S., Turolla R., 2006, MNRAS, 366, 727 